\title{
REVISIONES
}

\section{USO DE FUENTES DOCUMENTALES HISTORICAS QUE FAVORECEN LA INVESTIGACION FORMATIVA. EL CASO DE LOS SEMILLEROS DE INVESTIGACION*}

Use of historic documentaries that stimulate formative research.

The case of undergraduate research seedbeds

\author{
Carmen García \\ Historiadora y magíster en Educación, Universidad de Antioquia, Instituto de Educación Física, \\ Grupo de Investigación Estudios en Educación Corporal, Medellín, Colombia. \\ Teléfonos: (4)5381150 - (4)2199266. E-mail: carmenemiliagarcia@yahoo.es
}

\section{Resumen}

El artículo Uso de fuentes documentales históricas que favorecen la investigación formativa. El caso de los semilleros de investigación, surge de los resultados de la investigación "Recuperación de fuentes documentales para la historia de las prácticas pedagógicas del cuerpo escolarizado en la primera mitad del siglo XX en Medellín" y de la experiencia como orientadora de semilleros de investigación en el Instituto de Educación Física. La investigación formativa en los semilleros se favorece mediante las estrategias pedagógicas de la relación con el pasado mediado por el trabajo de elaboración de fuentes, de la pregunta como dispositivo de sensibilización, del principio investigativo del asombro y de la experiencia de la lectura.

Palabras clave: fuentes históricas, investigación formativa, semilleros de investigación, Educación Física.

\section{Summary}

The article, Use of Historic Documentaries that Stimulate Formative Research: The Case of Undergraduate Research Seedbeds, arises from the results of the research, "Retrieval of Documentary Sources for the History of Pedagogical Practices of the Teaching Body in the First Half of the Twentieth Century in Medellín", and from this experience as a guide for research seedbeds at the Physical Education Institute. Formative research in the seedbeds is stimulated by pedagogical strategies that relate to the past through preparation work of sources, of questions as devices for raising awareness, of amazement as a research principle, and of the experience of reading.

Key words: historical sources, formative research, research seedbeds, Physical Education.

\footnotetext{
* Proyecto financiado por la Vicerrectoría de Investigaciones y el Instituto de Educación Física de la Universidad de Antioquia.
} 


\section{INTRODUCCION}

La tarea en este artículo será hacer un tejido comprensivo entre el espacio formativo del semillero de investigación y la práctica pedagógica de investigación formativa, mediada por el uso de fuentes documentales históricas. Los análisis que se plantean encuentran una ejemplificación en las instituciones educativas del pregrado universitario, y podrán eventualmente delinear el porvenir de ciertas prácticas educativas encaminadas a pensar. El lugar desde el cual escribo es la universidad, pues es el lugar donde se hace más acerca de ciertas prácticas educativas, y creo que debería también pensar más en ellas. El oyente al que me dirijo es aquel que ha reflexionado sobre los problemas de la educación y sus prácticas pedagógicas. También el lugar está en el desarrollo del proyecto de investigación Recuperación de fuentes documentales para la historia de las prácticas pedagógicas del cuerpo escolarizado en la primera mitad del siglo XX en Medellín y en mi experiencia como orientadora de semilleros de investigación en el pregrado de Educación Física.

El proyecto se propuso identificar y describir las fuentes documentales que se encuentran en los diferentes archivos de la ciudad, diseñar una herramienta informática que permita la conservación, concentración y difusión de las fuentes y plantear una estrategia pedagógica de las fuentes documentales históricas que favorecen procesos de investigación formativa.

Las fuentes de información primarias estaban en los diferentes archivos de la ciudad de Medellín, de manera dispersa y desordenada. Nos dimos a la tarea de identificar aquellos lugares donde pudiéramos encontrar la información histórica que diera cuenta de nuestro interés. Los lugares identificados para tal fin fueron: Sala de Prensa y Sala de Patrimonio Documental de la Universidad de Antioquia; Archivo Histórico de Medellín; Archivo Histórico de Antioquia; Sala Antioquia de la Biblioteca Pública Piloto; la Normal de Medellín; Colegio la Salle. La información recogida en los archivos, a partir de fotografía y luego trascripción de información, se consignó en una ficha de recolección de información.

Una vez se han consignado las fichas en un programa de computador, se procede a describir de manera provisional unidades de relación con respecto al problema de investigación. Las unidades de clasificación que hasta la fecha se tienen son: educación de los sentidos, educación física, disciplinamiento del cuerpo, educación, higiene, raza, urbanidad, excursiones-paseos, anormalidad corporal, juegos, niño-joven, escultismo, alimentación.

Ahora, el semillero de investigación es un espacio de formación alternativo al plan de estudios, se ocupa del tema del cuerpo con perspectiva educativa fundamentado en la historia, el arte, la filosofía y la vida cotidiana, entre otros. Este es el lugar donde implementa el uso de las fuentes documentales históricas que favorecen la investigación formativa. El semillero está conformado por estudiantes y profesores interesados en la investigación formativa, con la finalidad de hacer de ellos sujetos críticos y reflexivos frente al diario vivir, soportado en el componente corporal a partir de la investigación. El semillero materializa sus actividades a partir de la lectura, la conversación, la escritura, la asistencia a conferencias y eventos académicos y culturales, la organización de eventos, la construcción de proyectos conjuntos y demás propuestas de intervención reflexiva. La metodología utilizada en el semillero es la tríada maestro-libro-estudiante. El maestro 
no es la inteligencia superior que traduce a las inteligencias de los estudiantes lo que dicen los libros. El maestro propicia el encuentro entre el libro y los estudiantes y retira de manera voluntaria su inteligencia del juego intelectual. Lo que el maestro pone en esta tríada es su propia experiencia, pero no mostrará lo que sabe, mostrará lo que ha escuchado del texto, así como los estudiantes mostrarán también lo que han logrado escuchar. Esta vía se puede llamar la de la confianza en la capacidad intelectual de todo ser humano y además se le persuadirá al estudiante a usar su propia inteligencia, a tener conciencia de su capacidad intelectual y decidir sobre su uso.

\section{USO DE FUENTES DOCUMENTALES HISTORICAS QUE FAVORECEN LA INVESTIGACION FORMATIVA}

\section{LA RELACION CON EL PASADO MEDIADA POR EL TRABAJO DE ELABORACION DE FUENTES Y EN PERSPECTIVA DE UNA RAZON SENSIBLE}

Las iniciativas intelectuales que se desarrollan en el semillero de investigación se dan en perspectiva de una razón sensible y obedecen a pequeñas razones momentáneas que no siempre corresponden con las razones institucionalizadas y dominantes. En los semilleros hay una oportunidad de asociar la práctica del acto de conocer y al mismo tiempo captar las pulsiones vitales de tal acto. El trabajo intelectual consiste en captar la vida que anima las iniciativas de estudio de los estudiantes, en valorar el mundo que los rodea, el mundo en que "están". Es captar también la sensibilidad que los anima y les permite cierta relación con el mundo y a partir de allí, como de una fuente, ponerse en camino y escuchar los intereses y las necesidades de conocimiento que los estudiantes tienen, captar el punto nodal a partir del cual se va a crecer intelectualmente y evitar delimitar desde afuera, recibir impulsos que no corresponden con lo que se es. Pero este ejercicio de comprensión demanda una mirada crítica sobre lo que se ha considerado como verdad y analizar el arraigamiento dentro del medio social y cultural en el cual se ha vivido.

Desde la perspectiva de la razón sensible, se acoge la propuesta de Jacques Rancière en su libro "El Maestro Ignorante", sobre la ausencia del maestro explicador. Los semilleros de investigación pueden prescindir del maestro explicador, pero no por ello del maestro. Se propone acá una tríada metodológica válida para los intercambios que se suscitan al interior del semillero: maestro-libro-estudiante. El maestro no es la inteligencia superior que traduce a las inteligencias de los estudiantes lo que dicen los libros. El maestro propicia el encuentro entre el libro y los estudiantes y retira de manera voluntaria su inteligencia del juego intelectual. A decir de Rancière (2002: 47) "la materialidad del libro pone a dos espíritus a una distancia que los mantiene como iguales, mientras que la explicación es aniquilación del uno por el otro".

Las iniciativas intelectuales de los integrantes del semillero comienzan en el ejercicio reflexivo de plantear un problema actual e intentar resolverlo a partir de la investigación histórica. Este ejercicio comienza con la selección de un tema, el planteamiento de un problema, las hipótesis, la identificación de los elementos que lo constituyen y la localización y selección de unas fuentes pertinentes. Con este comienzo se da inicio a la elaboración, transformación o tratamiento de las fuentes. Este movimiento de elaboración de materiales, de transformación de los documentos en otra cosa, a decir de Silva (2007, 
p. 60), "comienza desde el momento mismo en que se ingresa en un archivo para tratar de convertir un conjunto de documentos en el 'archivo de un problema', pues seleccionar unas fuentes es proceder a redistribuir un espacio, ya que en la investigación histórica todo comienza con 'el gesto de poner aparte, de reunir, de convertir en 'documentos' algunos objetos repartidos de otro modo, tal como lo señalaba Michel de Certeau (1993, p. 86), quien insistía en que había necesidad de 'desterrar los documentos', de alejarlos del sistema de relaciones habituales en que habían funcionado de "retirarlos" de la práctica 'para convertirlos en objetos abstractos de un saber', pues (...) lejos de aceptar los 'datos', el [historiador] mismo los forma. El material es creado por acciones concertadas que lo distinguen en el universo de su uso, que lo buscan también fuera de las fronteras de su uso y que lo destinan a un nuevo empleo coherente".

¿Y qué es un documento? Es un vestigio, un testimonio indirecto (cosas vistas por otros), un legado establecido y seleccionado antes de él y en relación con problemas por completo diferentes a los que él ahora quiere estudiar. El investigador se encuentra con un proceso de selección de fuentes, donde se ignoran los criterios que organizaron su clasificación o los azares que permitieron su conservación (Silva, 2007, p. 53). El documento es toda fuente de información de donde se extrae algo para el conocimiento del pasado humano, considerado desde el ángulo de la pregunta que se ha planteado (Marrou, 199, p. 62).

¿Cómo se produce el documento? A partir del gesto de poner aparte, de reunir unos objetos repartidos de otro modo. Por el hecho de recopilar, transcribir o fotografiar dichos objetos cambiando a la vez su lugar y su condición (De Certeau, 1993, p. 85). La elaboración de la fuente documental pertinente también incluye el planteamiento de una pregunta, porque es la manera en que un documento habla a los contemporáneos. La pregunta tiene forma de cuestionario, y los documentos sólo hablan cuando sabemos interrogarlos y tenemos de entrada una dirección. De dónde emerge el cuestionario: en ocasiones por las afirmaciones o dudas que las experiencias anteriores inscribieron oscuramente en el cerebro, por la tradición, el sentido común, es decir, con demasiada frecuencia, por los prejuicios comunes (...). "Por supuesto, esta selección racional de preguntas tiene que ser sumamente flexible, susceptible de recoger a lo largo del camino una multitud de elementos nuevos y abrirse a todas las sorpresas, tal como un imán que desde el comienzo atrae las limaduras del documento. El explorador sabe de antemano que no seguirá punto por punto el itinerario que se había fijado. Sin embargo, de no tenerlo, correrá el riesgo de errar eternamente a la aventura" (Bloch, 2001, p. 87).

La pregunta de investigación va enmarcada dentro de un uso adecuado de la teoría o perspectiva teórica, y es el primer paso en el método de trabajo con las fuentes convertidas en documentos. La teoría permite producir descripciones analíticas, es decir, guía la producción de análisis, de manera que se articule el material que se examina (fuente documental) con unas categorías de análisis. Las categorías de análisis de ponen a funcionar, sometiendo el material de archivo a formas iniciales pero distintivas de elaboración: la comparación, la contrastación, el examen de ejemplos que van en direcciones opuestas, etc., a la luz de hipótesis de trabajo (Silva, 2007, p. 70).

El segundo paso del método de trabajo en el ejercicio de investigación histórica consiste en transformar el documento en historia. Se trata de cambiar una cosa, que tenía ya su condición y desempeñaba su papel, en otra cosa que funcione de una manera distinta. 
Así pues, no se puede llamar "investigación" al estudio que adopta pura y simplemente las clasificaciones de ayer, que, por ejemplo, "se mantiene" en los límites trazados por la serie $\mathrm{H}$ del archivo, y que por lo tanto no define un campo objetivo propio (De Certeau, 1993, p. 87).

Al documento transformado en historia le concierne investigarlo, trabajarlo desde el interior y elaborarlo. Este trabajo consiste en definir y describir unidades de relación con respecto al problema de investigación. No es ya la materia inerte que sirve para reconstruir que los hombres han hecho o dicho. La historia es menos memoria y pasa a ser "textos más elaborados, más sofisticados, cargados de ideología; relatos de acontecimientos, de crónicas, de reportajes; es decir, de teorías sobre el orden del mundo" (Duby, 1988, p. 39).

\section{LA PREGUNTA COMO DISPOSITIVO DE SENSIBILIZACION}

La sensibilidad es la facultad de las personas de estar en relación con el mundo. Las personas establecen relación con los objetos desde su sensibilidad y ésta es con un interés de conocer, de obtener placer, de jugar. La experiencia se constituye en la producción de relaciones que se establezcan entre las instituciones y las personas, mediada por la sensibilidad. Atender más a los acontecimientos particulares de las personas en relación con el mundo y de ahí posibilitar la producción de nuevas experiencias y no tanto imitar las relaciones establecidas. Las personas podrán producir sentido con su experiencia y conformarán sus maneras de relacionarse y de hacerse visible para sí mismas. Las personas, entonces, no se someten a las experiencias establecidas de antemano, sino que van más allá y las rebasan y de esta manera se afectan en lo propio. Afectarse en lo propio significa que cada momento de su experiencia es un acontecimiento que vale por sí mismo, donde tiene valor lo heterogéneo y lo paradójico y se abre un campo donde las relaciones varían y pueden ser de otra forma.

Cada persona puede construir sentido a partir de su propia experiencia, de su sensibilidad individual que las configura como tales. Así, se verán formas diferentes de percibir el mundo, el otro y de darle sentido a sí mismos. Lo que está en juego en la experiencia como sensibilidad son las formas de darle curso a lo que les ocurre a las personas cuando se relacionan con el mundo y hace posible mirar la realidad y hacer variaciones sobre ella y sobre las maneras de habitarlo. Las variaciones que se puedan generar a partir de una experiencia sirven para hacer una composición con lo que le pasa a las personas.

La pregunta es la actitud vital de base para que las personas establezcan relación con el mundo desde la sensibilidad y es la condición inicial de toda comprensión. Mientras existan seres que pregunten, la comprensión del mundo será dinámica. Las preguntas movilizan procesos de pensamiento en los que se duda aun de la propia duda. Para comprender lo que significa pensar es preciso traer del griego el légein, que puede entenderse como "poner", "disponer", "estar echado", poner-se en camino. Si pensamos algo es que lo meditamos, que lo ponemos a consideración, lo retomamos. Los interrogantes se formulan y desarrollan a partir de contextos históricos particulares. Enseñar a preguntar, a cuestionar la propia vida en sus múltiples dimensiones, a dudar de lo que se presenta con una claridad aparente y sospechosa, interrogar los propios 
criterios y las propias motivaciones del hacer, preguntar con el ánimo creador del artista y responder de modo que estén abiertas nuevas preguntas (Soto, 2007).

El ejercicio de la pregunta evita que los acaeceres del mundo se nos presenten sin relaciones; en este sentido se aboga por el principio investigativo del asombro, el cual suscita la pregunta de por qué las cosas son como son y en la elaborada respuesta rompe con el mundo estéril, hecho lo cual se abre un mundo sugestivo hirviendo en interrogantes. El asombro rompe con el círculo de adecuar lo buscado a lo encontrado, abre abanicos y oxigena el ambiente. El rol del maestro en este ejercicio de la pregunta es discreto, él es el guía, pero "no verificará lo que ha encontrado el alumno, comprobará lo que ha buscado" (Rancier, 2002, p. 45).

\section{PRINCIPIO INVESTIGATIVO DEL ASOMBRO}

El principio investigativo del asombro lo encuentro ejemplificado en José Arturo Muñoz (1992, p. 40) de manera iluminadora para la práctica de la investigación formativa. El autor dice que un hecho presentado sin sus relaciones no es más que un hecho aislado, frío, inerte e inmóvil, no nos informa ni aclara nada más allá de la propia obviedad. La frase: "Pedro pasó por aquí,", no nos permite avizorar nada significativo acerca del conocimiento que sobre Pedro tenemos, ni del motivo de su paso y mucho menos del hombre Pedro y del conjunto de circunstancias que lo rodean y lo hacen congénere vivo, contradictorio y majo.

Los acaeceres del mundo se desvanecen ante la mirada imparcial y gélida que pretende hacer aparecer la llamada comúnmente "realidad" como algo que está ahí, pues no podemos percibir algo de verdadera valía si no hemos relacionado ese algo con una gama amplia y en muchas ocasiones intrincada de sucesos extraídos de la fragua de los conceptos. Concepto de especulación: hace referencia a la posibilidad teórica, de indicar la valía del pensamiento frente a los sucesos. Sin posibilidad de asombro la vida se convierte en estéril desierto, sin tener siquiera la posibilidad de cosechar las tunas, ni de apreciar sus costas. Sobre los hombres grises del libro MOMO, de Michel Ende, que prohibían el pensamiento y lo canjeaban por la producción impersonal, masificada y controlada del todo por cada minuto y segundo del tiempo de los hombres dedicado a producir. Este control sobre el pensamiento utilizado por las formas de poder restrictivo ocasiona, a la postre, que las construcciones culturales del mundo se desvanezcan o se ignoren y el hombre se vea obligado a debatirse entre la argumentación insulsa y la vanalidad de una vida carente de significado. Los personajes fundadores de un mito, o de una religión, o de una forma de arte, o de cualquier obra humana, tienen el sentido y la dirección que hace gala a su visión asombrosa; pero quienes llegan posteriormente y no lo redescubren sino lo ignoran o lo redescubren acríticamente, no encuentran las relaciones de estas expresiones y pierden así el significado, el asombro, es decir, su complejidad.

Es importante resaltar que sólo se encuentra lo que se busca previa concepción de lo buscado, así esa previa concepción en ocasiones parezca diluida: la capacidad de asombro. 


\section{LA EXPERIENCIA DE LA LECTURA}

La noción de experiencia que se aborda en este escrito se apoya en los trabajos del profesor español Jorge Larrosa. Es importante esta visión de la experiencia porque ubica su análisis en los espacios de interacción pedagógica, asunto que es de suma validez para nuestras intenciones educativas. Las posibilidades de la experiencia están en relación con lo que el autor llama exterioridad, reflexión y pasión. La cuestión de la exterioridad hace referencia a los acontecimientos que suceden y que no dependen directamente de las personas, que no son resultado "de mis palabras, ni de mis ideas, ni de mis representaciones, ni de mis sentimientos, ni de mis proyectos, ni de mis intenciones, que no depende ni de mi saber, ni de mi poder, ni de mi voluntad". Son las cosas que pasan ante mí (Larrosa, 2006, p. 44). El asunto de la reflexión, esta vez, sí está en relación con eso que me pasa. Es decir, el lugar de la experiencia soy yo. "Es en mí (o en mis palabras, o en mis ideas, o en mis representaciones, o en mi saber, o en mi poder, o en mi voluntad) donde se da la experiencia, donde la experiencia tiene lugar. El acontecimiento me afecta a mí, tiene efectos en mí, en lo que soy.

Es un sujeto capaz de dejar que algo le pase. Se trata de un sujeto abierto, sensible, vulnerable, expuesto. No hay experiencia en general, la experiencia siempre es de alguien. El sujeto hace la experiencia de su propia transformación, de ahí que la experiencia me forma y me transforma" (Larrosa, 2006, p. 45). La pasión, como posibilidad de la experiencia, supone "también que algo pasa desde el acontecimiento hacia mí, que algo viene hacia mí, que algo me viene o me ad/viene. Ese paso, además, es una aventura y, por tanto, tiene algo de incertidumbre, supone un riesgo, un peligro. Si la experiencia es "eso que me pasa", el sujeto de la experiencia es como un territorio de paso, como una superficie de sensibilidad en la que algo pasa y en la que "eso que me pasa" al pasar por mí o en mí, deja una huella, una marca, un rastro, una herida (Larrosa, 2006, p. 46).

Ahora bien, la experiencia de la lectura en este contexto descrito anteriormente alude a la relación que el investigador pueda establecer con los textos o las fuentes documentales. Lo importante no es el texto, sino la relación con el texto, lo que nos pasa con la lectura. Es hacer del ejercicio de la lectura un juego, donde el lector se pone en juego él mismo, donde está en juego su propia subjetividad, un lector que sale al encuentro con el texto para reflexionar y que esté dispuesto a que los textos le digan cosas, a transformarse. A decir de Jorge Larrosa (2006, p. 49) "la experiencia es una relación en la que algo tiene lugar en mí. En este caso, que mi relación con el texto, es decir, mi lectura, es de condición reflexiva, vuelta para adentro, subjetiva, que me implica en lo que soy, que tiene una dimensión transformadora, que me hace otro de lo que soy. Por eso, después de la lectura, yo ya no soy el mismo que era, ya no puedo mirarme impávido al espejo".

Se alude aquí a una experiencia de la lectura, del conocimiento y pensamiento con respecto al pasado. De la relación presente pasado, donde está en juego nuestra relación con el pasado, mediada por la fuente documental histórica como texto. Lo importante en esta noción de experiencia que nos propone Larrosa es tomar en cuenta que una relación con la lectura, en nuestro caso con el documento, nos puede ayudar en el acto educativo, a transformar las maneras como nos hemos referido al pasado. Pero también y muy importante, una relación como la que sugiere este tema a investigar, la pregunta como dispositivo de sensibilización, la elaboración de la fuente documental, el principio investigativo del asombro y experiencia de la lectura, posibilita en los jóvenes en el 
ejercicio investigador, formar o transformar su propio lenguaje, los invita a hablar por sí mismos, a escribir por sí mismos, a pensar por sí mismos.

En la experiencia vivida de un semillero de investigación hay momentos en que cuentan menos los compromisos institucionales de producción de conocimiento y de seguimiento a indicadores de gestión académica. Asimismo, hay momentos en que importa menos la formación de la persona en cuanto a la sensibilidad que se tenga con el conocimiento, respecto para con la pregunta, a minimizar la figura del maestro explicador. Estos dos aspectos son característicos en el contexto de los semilleros de investigación, mediante la denominación de proxemia. Para el análisis de esta proxemia, hay que estar atentos no sólo al componente relacional que se da entre los semilleros de investigación y la vida institucional y social, a los individuos en relación, sino también a lo que fija a las personas a su entorno, a su ciudad, a un territorio.

Las comunidades de investigación formativa expresadas en los semilleros de investigación descansan en la doble polaridad de un elogio de la razón sensible y un imperio de la razón abstracta, lo que no ha impedido, hasta ahora, la proliferación de semilleros. Los semilleros, de hecho, son de diferentes órdenes, pero desde el punto de vista estructural, es su diversidad la que asegura su perennidad. Sin embargo, es necesario efectuar un cambio de óptica, donde se ponga el acento en una proxemia de acercamiento y menos en la proxemia de la distancia entre la razón sensible y la abstracta.

Esta propuesta de una especie de fraternidad surgida de la proximidad que representa la potencia que tienen los semilleros de pensarse como comunidades de afección por el conocimiento, de equidad con el saber e indagar por lo que les pasa en la vivencia cotidiana, que no consiste en pensar lo que sea, sino en hacer aquello que agota la definición del ser, en definitiva, un ser que se piensa.

No se alude acá al equilibrio armonioso entre la razón sensible y la razón abstracta, se alude más a la proxemia del acercamiento, que permite observar, comparar, combinar, hacer y atender cómo se han hecho las cosas. Aplicar una de las etimologías imaginarias de Cratilo: "el hombre, el anthropos, es el ser que examina lo que ve, que se conoce en esa reflexión sobre su acto" (Rancière, 2002: 52). La vuelta sobre lo que se ha hecho en los semilleros en cualquiera de las dos razones, es atención y reflexión sobre los actos intelectuales. La proxemia del acercamiento persuade para que los integrantes de los semilleros no dejen de vivir experiencias que les inciten a pensar en términos de razonar, de calcular o de argumentar, sino que "pensar es dar sentido a lo que somos y a lo que nos pasa" (Larrosa, 2003: 166).

\section{BIBLIOGRAFIA}

Bloch, M. (2001). Apología para la historia o el oficio de historiador. México: Fondo de Cultura Económica.

De Certeau, M. (1993). La escritura de la historia. Colombia: Universidad Iberoamericana: Departamento de Historia.

Duby, G. (1988). Diálogos sobre la historia. Conversaciones con Guy Lardreau. Madrid: Alianza Editorial. 
Foucault, M. (1999). La arqueología del saber. México: Siglo Veintiuno Editores.

Larrosa, J. (2006). ¿Y tú qué piensas? Experiencia y aprendizaje. Revista Educación y Pedagogía. Separata, Medellín, Universidad de Antioquia. Facultad de Educación, 43-67.

Larrosa, J. (2003). Entre las lenguas. Lenguaje y educación después de Babel. Barcelona: Laertes.

Maffesoli, M. (1997). Elogio de la razón sensible. Una visión intuitiva del mundo contemporáneo. Barcelona: Paidós.

Maffesoli, M. (1990). El tiempo de las tribus. Barcelona: Icaria.

Mandoki, K. (2006). Prácticas estéticas e identidades sociales. Prosaica Dos. México, Siglo XXI.

Marrou, H.-I. (1999). El conocimiento histórico. España: Idea Universitaria.

Muñoz, J. A. (1992). El oficio de investigar o el arte de auscultar las estrellas. Bogotá: CORPRODIC.

Nietzsche, F. (1980). Sobre el porvenir de nuestras escuelas. Barcelona: Tusquets Editores.

Rancier, J. (2002). El maestro ignorante. Barcelona, Laertes.

Silva, R. (2007). A la sombra de Clío. Diez ensayos sobre historia e historiografía. Medellín: La Carreta Histórica.

Soto, G. (2007). Lineamientos Curriculares para la Enseñanza y el Aprendizaje de la Filosofía en la Educación Media. Medellín: Universidad Pontificia Bolivariana.

Veyne, P. (1984). Cómo se escribe la historia. Foucault revoluciona la historia. Madrid: Alianza Editorial. 
